\title{
Textile-based wearable sensors for assisting sports performance
}

\author{
Shirley Coyle ${ }^{1}$, Deirdre Morris ${ }^{1}$, King-Tong \\ Lau $^{1}$, Dermot Diamond ${ }^{1}$ \\ ${ }^{1}$ Clarity: The Centre for Sensor Web Technologies, \\ National Centre for Sensor Research, Dublin City \\ University, Dublin 9, Ireland \\ shirley.coyle@dcu.ie,deirdre.morris@dcu.ie, \\ kim.lau@dcu.ie,dermot.diamond@dcu.ie
}

\begin{abstract}
There is a need for wearable sensors to assess physiological signals and body kinematics during exercise. Such sensors need to be straightforward to use, and ideally the complete system integrated fully within a garment. This would allow wearers to monitor their progress as they undergo an exercise training programme without the need to attach external devices. This takes physiological monitoring into a more natural setting. By developing textile sensors the intelligence is integrated into a sports garment in an innocuous manner. A number of textile based sensors are presented here that have been integrated into garments for various sports applications.
\end{abstract}

Keywords - textile sensors, wearable technology, sweat analysis, physiological monitoring

\section{INTRODUCTION}

Wearable sensors can be used to monitor the body's physiological response to exercise and also the kinematic aspects of performance. To monitor this in a natural way there is a need for integrated sensors that are comfortable, wearable and straightforward to use. Textile based sensors which are compatible with textile manufacturing processes are essential for such technology to become accessible. Making the fabric itself the sensor can augment garment functionality while still maintaining the normal tactile properties of the garment.

Here we present textile based sensors suitable for monitoring the wearer during exercise. The first sensor discussed is a $\mathrm{pH}$ sensor which collects and analyses sweat in real-time. This is a novel approach to chemical sensing on fabric. The following sensors discussed are piezoresistive sensors which respond to body movement such as breathing, joint motion and foot plantar pressure. Fabric circuitry and integration issues are addressed for all of these sensors. Typical responses for each sensor type are presented and potential applications are proposed.

\author{
Niall Moyna ${ }^{2}$ \\ ${ }^{2}$ Clarity: The Centre for Sensor Web Technologies, \\ School of Health and Human Performance, Dublin \\ City University, Dublin 9, Ireland \\ niall.moyna@dcu.ie
}

\section{TEXTILE SENSORS}

\section{A. Body fluids, chemical sensors}

Rehydration is an importance factor in athletic performance [1]. Not only is it important to replace fluids but also to replace electrolytes that are lost through sweating. As part of the Biotex project, an EU funded project [2], a fabric based system has been developed to analyse sweat during exercise[3, 4]. A fabric channel is dyed with a $\mathrm{pH}$ sensitive indicator which has a colorimetric response. LEDs positioned above the channel are used to detect colour change, using a paired LED approach[5]. This approach makes it possible to collect and analyse sweat directly in real-time, using moisture-wicking fabrics that are typically used in sports clothing. By analyzing the signals in real-time it is possible to give immediate feedback to the coach or athlete. Information regarding the composition and amount of sweat lost is important for ensuring adequate hydration levels are met. This technology has the potential to prescribe individualized rehydration strategies that would be of particular benefit during acclimatization to temperature and humidity in different countries. The current system design is shown in Figure 1, the patch dimensions are $60 \times 40 \times 7 \mathrm{~mm}$ and it is attached to the lower back using a waistband. A wireless microcontroller

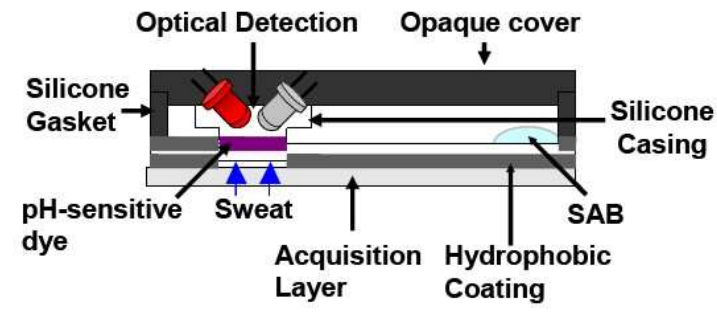

Figure 1. Textile based wearable patch for sweat analysis 


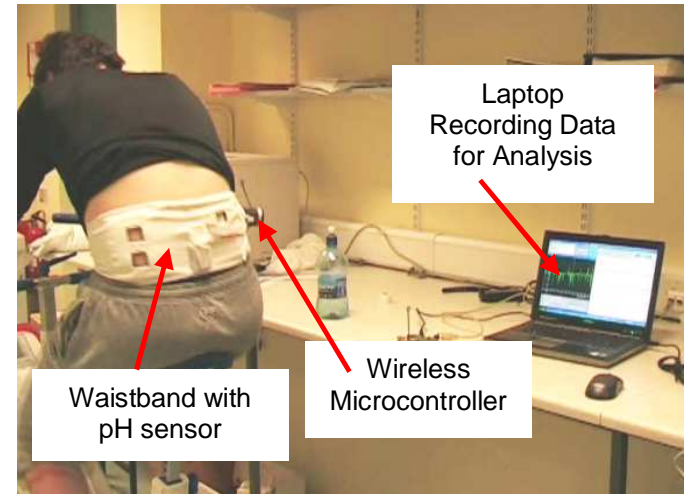

Figure 2. Experimental set-up for sweat $\mathrm{pH}$ sensor

(Crossbow mica2dot mote or custom-made device provide by CSEM[6]) is used to control the optical detection performed by the LEDs. The detected light intensity is sampled at a rate of $0.2 \mathrm{~Hz}$ and transferred wirelessly to a nearby laptop for analysis as shown in Figure 2.

\section{B. Physiological monitoring}

Breathing pattern is a straightforward indicator to assess how hard the body is functioning. Fast breathing rates accompany a heightened heart rate and give us an indication of perceived effort. A wearable unobtrusive sensor would allow the measurement of breathing patterns over a number of training sessions to assess performance and recovery to exercise. There are also times when slow, controlled breathing is required - for practices such as yoga and pilates [7]. In athletes respiratory muscle training has been shown to improve exercise tolerance and athletic performance [8]. Measuring breathing rates and patterns can provide useful biofeedback for such techniques. Improved breathing techniques are also beneficial to general heath and well-being and particularly useful to those affected by stress [9]. Breathing rates can be measured by detecting the expansion and contraction of the ribcage using fabric strain or pressure sensors [10, 11]. Modified foams coated with the conducting polymer polypyrrole(PPy) or loaded with carbon are encased within a pocket in a garment or chest-strap at the ribcage to detect such movements. When the ribcage expands during inhalation the foam is compressed and its conductivity increases [12]. When the foam is released due to exhalation the conductivity returns to baseline. Similarly this can be implemented with modified stretch knit fabrics. These sensors are easily incorporated into a garment design, and connected to via conductive threads. A drawing of a smart shirt with a lycra ${ }^{\circledR}$ PPy sensor is shown in Figure 3.

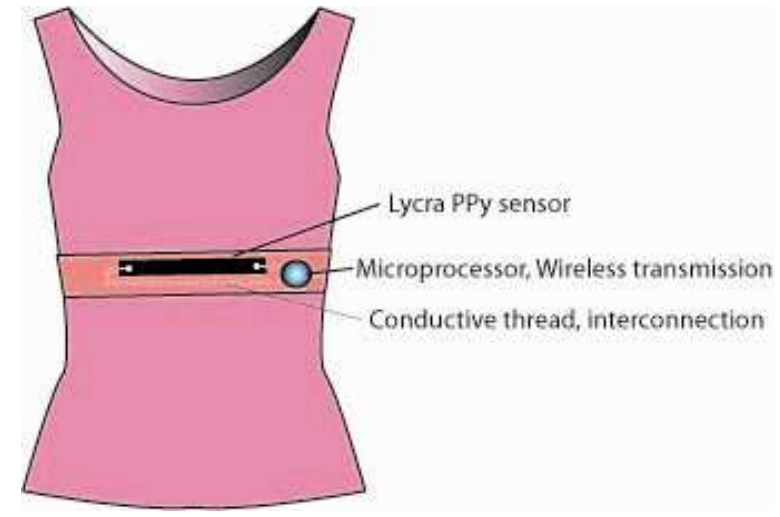

Figure 3. Shirt to measure breathing rate using fabric stretch sensor.

\section{Body kinematics}

Dynamic postural alignment is the basis for motor skills training[13]. Athletic movement does not rely on static posture but flowing dynamic movements. Good athletic posture is actually a series of postures linked together to produce efficient movement. Sports clothing which is usually close fitting is an ideal means of capturing the kinematics of an athlete. Feeding back this information to the athlete reinforces body awareness which may help to improve technique. The most common approach is assessment by the naked eye and video which may not always be practical. The watchful eye of coach and expensive video equipment may not be available whereas sports clothing is a universal necessity.

The fabric sensors described in the previous section can also be used to measure body kinematics. Joint movements can be measured using the fabric strain sensors while foot plantar pressures can be monitored by integrating the modified foam into shoe insoles. In this way dynamic information may be gleamed from the textile e.g. wrist flexion during racquet sports, knee bend during running or gait analysis during walking.

\section{FABRIC CIRCUITRY}

A control system is necessary to power the sensors and also to capture data and relay this information to the wearer in a productive manner. The advantage of using the conductive piezo-resistive materials is that they can be connected to directly using conductive thread. Bekaert stainless steel thread has been used and stitched by machine by placing the conductive thread in the lower bobbin case of a sewing machine. Issues of connecting conductive threads to brittle microcontroller components 


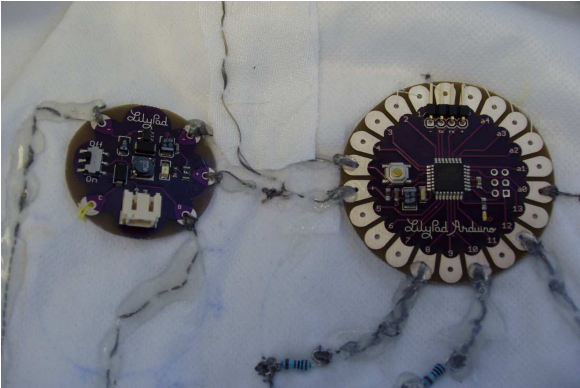

Figure 4. Lilypad arduino with power supply to connect polymer lithium battery.

have been addressed with a novel Arduino platform called Lilypad which has been designed especially for wearable applications[14]. Petals on the main circuit board contain holes which allow the conductive thread to be stitched in place to form a connection. This forms a secure connection avoiding the use of solder or crimped connections. The Lilypad system allows a dispersed modular structure through the garment. Modules to supply power, light sensors, temperature sensors and accelerometers are available in a similar circular petal design. Vibrotactile modules, LEDs and buzzers can be used to give instant feedback from the garment to the user in response to a particular stimulus. Such prompts may be used to improve the motor skills and posture during training. Figure 4 shows how the Lilypad mainboard is stitched in place by conductive thread and connected to a power supply module. The conductive threads have been insulated using a hot glue gun, although 3D fabric paint may also be used. With fabrics moving and being folded it is vital to ensure that short circuits do not occur. Another issue with stainless steel conductive threads is that the fibres tend to fray which can cause short circuits across components. This is another reason why it is imperative to properly insulate connections.

\section{RESUlTS}

\section{A. Sweat $p H$ sensor}

The sensor was calibrated using artificial sweat ranging from $\mathrm{pH} 4-8$. The results of three calibrations on the same sensor are shown in Figure 5. This illustrates that the sensor is sensitive to changes in $\mathrm{pH}$ of less 0.2 units and that the response is repeatable. The top cover incorporating the LEDs can be used for multiple exercise trials while the fabric layer containing the dye is easily replaced for each use. Each fabric patch is calibrated using $\mathrm{pH} 4$ and ph 8 following an exercise trial.

The response of the textile $\mathrm{pH}$ sensor during an exercise trial is shown in Figure 6. The sensor was placed on the subject's lower back during an hour-long exercise trial. The exercise involved indoor cycling at a selfselected pace. Reference measurements have been taken

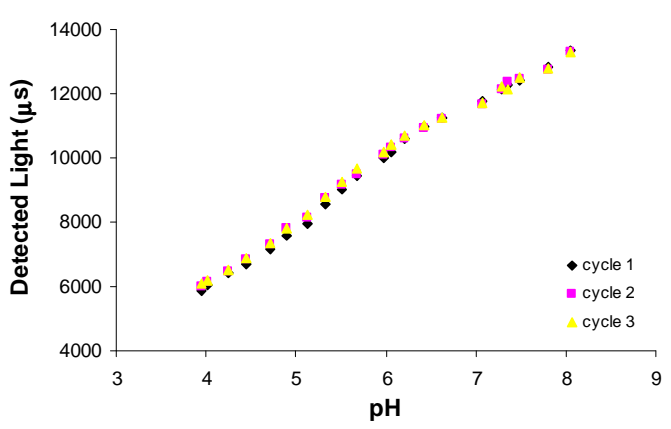

Figure 5. Calibration of the $\mathrm{pH}$ fabric sensor

using a flat surface $\mathrm{pH}$ electrode placed on the skin surface at five minute intervals. This was performed once adequate sweat was produced on the skin following a "priming" time as the subject warmed up. The results show very good correlation between the textile sensor and the reference $\mathrm{pH}$ measurements. There is a priming time involved which depends on the amount of time it takes for the subject to begin sweating and the time taken for the sweat to travel through the fabric channel and interact with the fabric sensor surface. Further testing is needed to establish the reasons for the change in $\mathrm{pH}$, as research in this area is unfortunately lacking due to the difficulty in sampling sweat. One hypothesis is that during exercise the sweat secreted at the skin is initially acidic due to reabsorption processes in the sweat duct [15]. However, as the subject continues to exercise, the rate at which sweat is excreted increases in order to regulate body temperature. This reduces the amount of time available for re-absorption processes and thus sweat $\mathrm{pH}$ increases along with electrolyte concentrations.

\section{B. Breathing rate sensor}

The response of the fabric sensor as the ribcage expands and contracts is shown in Figure 7. The sensor is easily integrated, with very simple circuitry. It is connected in series with a resistor as a straightforward voltage divider set-up, powered by the supply pins of the

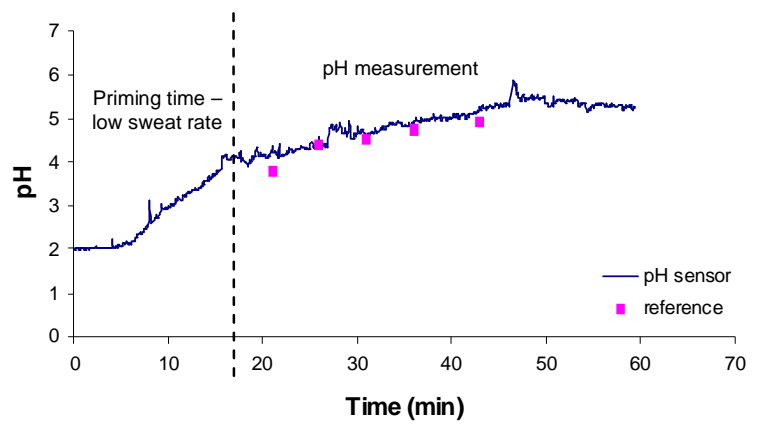

Figure 6. $\mathrm{pH}$ sensor reponse during an exercise trial 


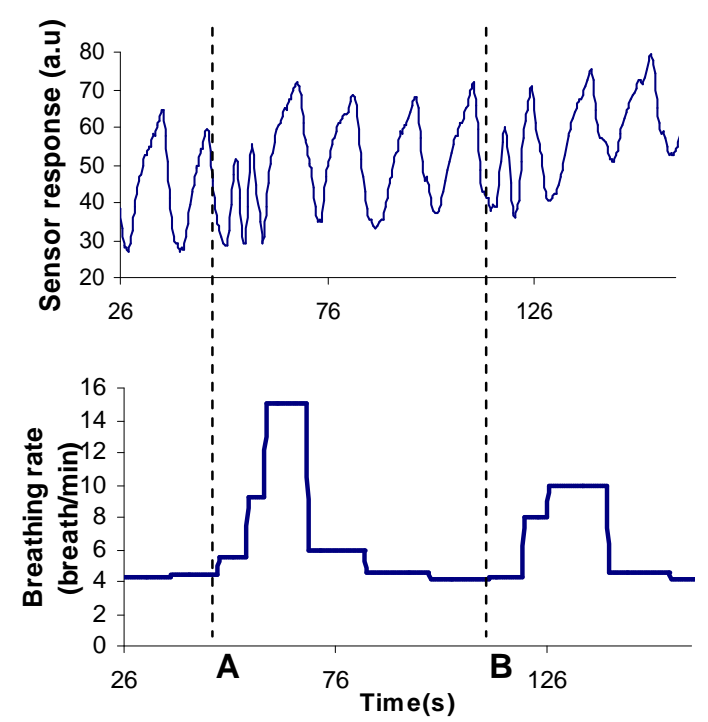

Figure 7. Breathing patterns detected using conductive foam sensor

Lilypad Arduino. The signal has been sampled at $2 \mathrm{~Hz}$. In addition to sampling the data, the Lilypad Arduino is programmed to detect peaks in the signal by calculating the slope at each sampling point and then finding points of inflection. The time taken between each point of inflection is used to calculate the breathing rate which is displayed in breaths/min as a function of time in Figure 7. It can be seen that at time A and B there is a shorter time between points of inflection and this is represented by an increased breathing rate.

\section{Joint flexion sensor}

The response of a fabric bend sensor integrated into a glove is shown in Figure 8. Carbon-loaded elastomer commercially available from WACKER Ltd (Elastosil LR $3162 \mathrm{~A} / \mathrm{B}$ ) has been coated onto the fingers of a glove. This approach has been demonstrated in the University of Pisa for measuring body kinematics [16]. The Arduino Lilypad has been used to collect the data using an analog input, sampled at $10 \mathrm{~Hz}$. The sensor response of the index finger is shown in Figure 8 as the fingers move from position 0 to 2 as demonstrated in Figure 9. This glove has been developed for rehabilitation applications based on the Fugel-Meyer Assessment of motor recovery after stroke. The Fugel-Meyer assesses various motor functions and scores them based on performance where 0 = cannot perform; $1=$ performs partially; $2=$ performs fully. Monitoring joint flexion as demonstrated here with the rehabilitation glove has the potential to automatically assess the patient's performance. This method of garment-based interactions would allow a person to be assessed from home on a continual basis with the remote supervision of a trained physical therapist. This approach

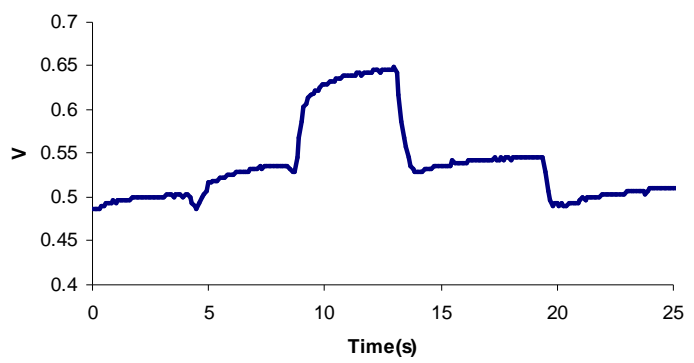

Figure 8. Response of CE sensor integrated into a glove during finger flexion (0), halfway extension (1) and full extension (2)

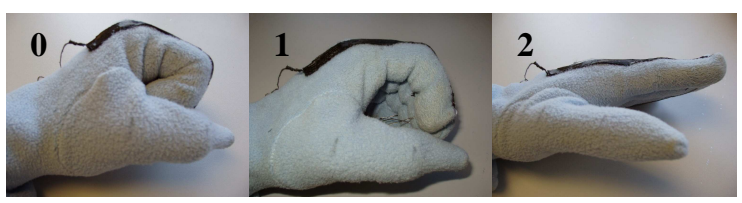

Figure 9. Hand position during finger flexion/extension exercise using the textile sensing glove

could be implemented to assess any joint depending on the movement of interest for various sports. Figure 10 shows the response of the sensor integrated into an elbow support. Repeated flexion of the elbow was performed, from straight arm to full flexion. The response shows good repeatability. Future work is needed to investigate the response at various angles of flexion. This approach has been demonstrated by the University of Wollongong with a conducting polymer sensor to assess knee bend during Australian football [17]. Consultation with coaches and physiotherapists is needed to identify movements of interests for particular sports to identify optimum placement for the sensors. This approach serves to improve performance while also reducing the risk of injury.

\section{DISCUSSION}

The sensors presented here provide a low-cost solution to physiological sensing with the added benefit of being

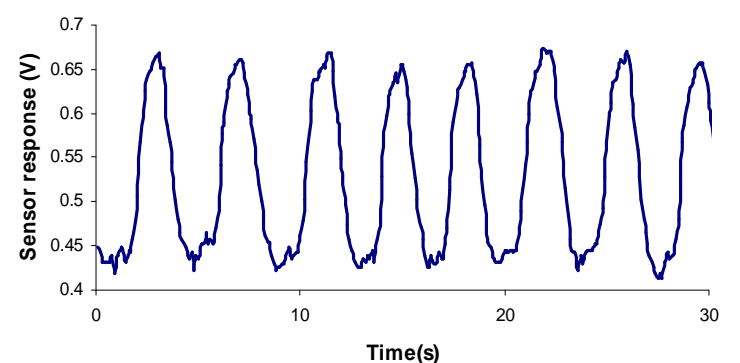

Figure 10. Response of elastomer sensor during elbow flexion and extension 
able to function in any setting where the wearer may choose to train. Not only is this valuable to the elite athlete but also for amateurs who wish get the most from their training regime and assess their progress. There is a need for wearable technologies that are comfortable to wear and also straightforward to use. This calls for a combined effort on one hand from the materials side in terms of developing functionalized fabrics and tackling integration issues, and also from the computer applications side to provide user-friendly interfaces. The world of human-computer interaction is evolving as technology becomes more and more ubiquitous. To make the technology accessible it must be easy to use e.g. a sports top which can collect all the physiological data, give feedback during the exercise while also saving the data for downloading later. It needs to fit in with the wearer's lifestyle and be hassle-free. The sports industry has a large market for such technologies, current technologies on the market include the Nike plus iPod [18] which captures running speeds and training times, Numetrex [19] manufacture a heart rate monitoring top, and Polar provide heart rate monitors, stride counters and GPS [20]. Another area where sensors are increasingly being used is interactive gaming, e.g. the Wii Fit by Nintendo [21] monitors balance for aerobic, strength and yoga training. This type of feedback could not only be useful for entertainment but also for rehabilitation purposes.

\section{CONCLUSIONS}

Textile based sensors enable technology to be integrated into clothing without affecting the look and feel of the cloth. Here we present two different types of textile based sensors - a fabric $\mathrm{pH}$ sensor to collect and analyse sweat and piezoresistive textiles to capture body movements. These have been integrated into clothing and shown to be useful for sports applications. Future work will characterize their response and assess functionality for specific applications. This will also require the development of suitable user interfaces that are straightforward and accessible.

\section{ACKNOWLEDGEMENT}

We gratefully acknowledge the financial support of the European Union (Biotex FP6-2004-IST-NMP-2) and Science Foundation Ireland (07-CE-I1147).

\section{REFERENCES}

R. J. Maughan, J. B. Leiper, and S. M. Shirreffs, "Rehydration and Recovery after Exercise," Sports Science Exchange, vol. 9, pp. 1-5 Supplement 62, 1996.

[2] $\quad$ http://www.biotex-eu.com, accessed Jan 2009.
[3] S. J. Morris and J. A. Paradiso, "Shoe-integrated sensor system for wireless gait analysis and real-time feedback," presented at Second Joint EMBS/BMES Conference, Houston, Texas, 2002.

[4] S. Coyle, Y. Wu, K. Lau, S. Brady, G. G. Wallace, and D. Diamond, "Bio-sensing textiles - Wearable Chemical Biosensors for Health Monitoring," presented at Body Sensor Networks 2007, Aachen Germany, 2007.

[5] K. T. Lau, W. S. Yerzunis, R. L. Shepherd, and D. Diamond, "Quantitative colorimetric analysis of dye mixtures using an optical photometer based on LED array," Sensors and Actuators B, vol. 114, pp. 819825,2006

[6] http://www.csem.ch, accessed Jan 2009.

[7] C. Gilbert, "Yoga and Breathing," Journal of Bodywork and Movement Therapies, vol. 3, pp. 44-54, 1999.

[8] A. Sheel, "Respiratory muscle training in healthy individuals: physiological rationale and implications for exercise performance.," Sports Medicine, vol. 32, pp. 567-581, 2002.

[9] M. S. Schwartz and F. Andrasik, Biofeedback: A Practitioner's Guide: Guilford Press, 2005.

[10] F. Carpi and D. De Rossi, "Electroactive PolymerBased Devices for e-Textiles in Biomedicine," IEEE Transactions on Information Technology in Biomedicine, vol. 9, pp. 295-318, 2005.

[11] S. Brady, B. Carson, N. Moyna, D. O'Gorman, and D. Diamond, "Body Sensor Network based on soft polymer sensors and wireless communications " Journal of Communications, vol. 2, pp. 1-6, 2007.

[12] S. Brady, D. Diamond, and K. T. Lau, "Inherently conducting polymer modified polyurethane smart foam for pressure sensing," Sensors and Actuators A, vol. 119, pp. 398-404, 2005.

[13] V. A. Gambetta, Athletic Development - The Art \& Science of Functional Sports Conditioning Human Kinetics Inc., 2007.

[14 http://www.arduino.cc/en/Main/ArduinoBoardLilyPad, accessed Jan '09.

[15] E. Fishberg and W. Bierman, "Acid Base Balance of Sweat," The Journal of Biological Chemistry, vol. 97, pp. 433-441, 1932.

[16] F. Lorussi, E. P. Scilingo, M. Tesconi, A. Tognetti, and D. De Rossi, "Strain Sensing Fabric for Hand Posture and Gesture Monitoring," IEEE Transactions on Information Technology in Biomedicine, vol. 9, pp. 372-381, 2005.

[17] B. J. Munro, T. E. Campbell, G. G. Wallace, and J. R. Steele, "The intelligent knee sleeve: A wearable biofeedback device " Sensors and Actuators B: Chemical, vol. 131, pp. 541-547 2008.

[18] http://nikeplus.nike.com/nikeplus/index.jhtml, accessed Jan 2009.

[19] http://www.numetrex.com/, accessed Jan 2009.

[20] http://www.polarusa.com/us-en/, accessed Jan 2009.

[21] http://www.nintendo.com/, accessed Jan 2009. 